\title{
Feasibility Study of Compton Scattering Enchanced Multiple Pinhole Imager for Nuclear Medicine
}

\author{
L. J. Meng, W. L. Rogers, N. H. Clinthorne, and J. A. Fessler
}

\begin{abstract}
This paper presents a feasibility study of a Compton scattering enhanced (CSE) multiple pinhole imaging system for gamma rays with energy of $140 \mathrm{keV}$ or higher. This system consists of a multiple-pinhole collimator, a position sensitive scintillation detector as used in standard Gamma camera, and a Silicon pad detector array, inserted between the collimator and the scintillation detector. The problem of multiplexing, normally associated with multiple pinhole system, is reduced by using the extra information from the detected Compton scattering events. In order to compensate for the sensitivity loss, due to the low probability of detecting Compton scattered events, the proposed detector is designed to collect both Compton scattering and non-Compton events. It has been shown that with properly selected pinhole spacing, the proposed detector design leads to an improved image quality.
\end{abstract}

Index Terms-Compton scattering enhancement, multiple pinhole.

\section{INTRODUCTION}

$\mathbf{P}$ ARALLEL hole and pinhole are by far the most often used collimation methods for nuclear medicine applications, although fanbeam and conebeam collimators are also applied [1], [2]. These collimators have the advantage of being nonmultiplexing and therefore every detected gamma ray defines a unique angular region, within which the gamma ray was generated or scattered. However, this uniqueness of information is achieved at the cost of losing all photons that do not fall into those possible paths defined by the collimator. Several mechanical collimators involving certain degrees of multiplexing have been studied in the past. These include the use of coded apertures, multiple pinholes, and rotating slits [3], [4], which allows a better raw detection sensitivity to be achieved. Unfortunately, this improvement is usually achieved at the expense of the amount of information conveyed by each detected photon. This tradeoff is similar for Compton cameras. The angular information carried by the detected photon is diluted into a conical surface. Furthermore the angular accuracy achievable is also limited by the effect of Doppler broadening and electronic noise, especially at lower energies [5]. It has been shown that at $140 \mathrm{keV}$, to compete with conventional gamma camera, the design of Compton camera may require an unrealistic amount of semiconductor material for the scattering detector to provide enough detected scattering events [6].

Manuscript received January 27, 2003; revised April 21, 2003

L. J. Meng, W. L. Rogers, and N. H. Clinthorne are with the Department of Radiology, University of Michigan, Ann Arbor, MI 48109 USA (e-mail: ljmeng@umich.edu).

J. A. Fessler is with the Department of Electrical Engineering and Computer Science, University of Michigan, Ann Arbor, MI 48109 USA.

Digital Object Identifier 10.1109/TNS.2003.817956

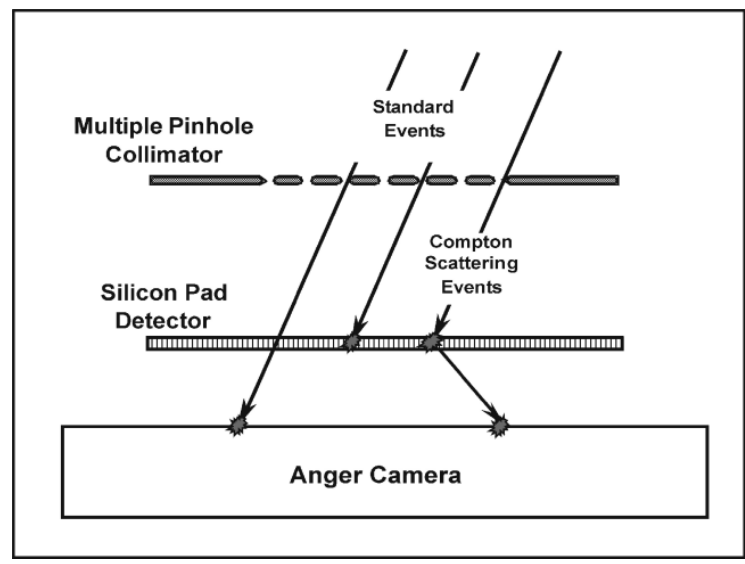

Fig. 1. Schematic of the Compton scattering enhanced multiple pinhole imaging system.

The idea of using combined mechanical and electronic collimations was previously proposed by Uritani et al. [7]. In their detector, a multiple parallel plate collimator was placed in front of a Compton camera. It reduces the effect of multiplexing, whilst maintains a relatively high open fraction on the collimator. The results showed an improved signal-to-noise ratio for imaging ${ }^{99 \mathrm{~m}} \mathrm{Tc}$ tracer. However, the raw detection sensitivity is limited by the relatively low probability of detecting photons through Compton scattering effect. In this paper, we present an alternative detector configuration based on the similar principle. It consists of a multiple pinhole collimator placed in front of a less optimized Compton camera. The choice of using multiple pinhole collimator offers a wide range of freedom in selecting the open fraction of the collimator and spatial coding scheme for different applications. The proposed imager is designed to collect events both Compton scattered between the two detectors and interact with either the first or the second detector only. Therefore, the raw sensitivity is not limited by the relatively low probability of detecting an incoming photon through Compton scattering. To evaluate the detector performance, we used the variance-resolution tradeoff curve calculated based on the maximum a posteriori (MAP) reconstruction methods [8], [9].

\section{THE DETECTOR CONCEPT}

The proposed detector system is essentially the combination of a Gamma camera using a multiple pinhole collimator and a Silicon scattering detector inserted between the Anger camera and the collimator, as shown in Fig. 1. For a detected Compton scattered event, the energy loss in the first detector and the locations of interactions on both detectors help to restrict the origin 
TABLE I

PARAMETERS USED IN THE SimUlation

\begin{tabular}{|c|c|c|c|c|}
\hline & First Detector & Second Detector & $\begin{array}{l}\text { Multiple Pinhole } \\
\text { Aperture }\end{array}$ & Object \\
\hline Total Size (mm) & $250 \times 250 \times 20$ & $400 \times 400 \times 6$ & $250 \times 250 \times 5$ & $96 \times 96 \times 48$ \\
\hline Pixel Size (mm) & $2.5 \times 2.5 \times 2.5 \mathrm{~mm}$ & $4 \times 4 \times 6$ & (Pinhole size) $\varnothing 1.5 \mathrm{~mm}$ & $3 \times 3 \times 3$ \\
\hline No. of pixels & $100 \times 100 \times 8\left(8 \times 10^{4}\right)$ & $10^{4}$ & $\begin{array}{c}\text { (No. of pinholes) } \\
9 \text { to } 225 \\
\end{array}$ & $32 \times 32 \times 16(16384)$ \\
\hline Z-location (mm) & -50 & -150 & 0 & $50 \sim 100$ \\
\hline Material & $\mathrm{Si}$ & $\mathrm{NaI}(\mathrm{Tl})$ & Tungsten & Air \\
\hline Energy Resolution & $\begin{array}{l}\text { Elec. Noise }(1 \mathrm{keV})+ \\
\text { Doppler Broadening }\end{array}$ & $10 \%$ at $140 \mathrm{keV}$ & -- & -- \\
\hline Energy threshold & $2.5 \mathrm{keV}$ & $20 \mathrm{keV}$ & -- & -- \\
\hline Notes & $\begin{array}{l}\text { 1. Doppler Broadening is } \\
\text { included. }\end{array}$ & $\begin{array}{l}\text { 1. Depth of interaction not } \\
\text { included. } \\
\text { 2. Resolution is equal to } \\
\text { pixel size. }\end{array}$ & $\begin{array}{l}\text { 1. Aperture penetration is } \\
\text { modeled. } \\
2 . \text { Photons scattered in } \\
\text { collimator ignored. }\end{array}$ & $\begin{array}{l}\text { 1. Object scattering and } \\
\text { attenuation ignored. } \\
\text { 2. Scattering in collimator } \\
\text { ignored. }\end{array}$ \\
\hline
\end{tabular}

of the photon to a broadened conical surface started from the first interaction location. This extra information not only enables one to say that the detected photon is from one of the pinholes in the collimator, but also assigns a different probability for each pinhole. The proposed detector system also makes use of those events that interact with either the first or the second detector only. The data containing both Compton scattered and non-Compton scattered events are used in list-mode image reconstruction. The Compton aperture is not designed for imaging by itself, but rather for restricting the already restricted angular uncertainty (through the mechanical aperture). It is possible to use a less optimized Compton camera whilst still provide useful extra information. For simplicity, we will use the term "CSE detector" for the proposed design and "MPH detector" for standard multiple pinhole detector. The key question that we would like to answer is "would the extra information provided by Compton scattering be sufficient for compensating the loss in the number of photons collected?"

\section{MeTHODS FOR COMPARISONS}

\section{A. Detector Simulation and Image Reconstruction}

The basic detector and source configurations used in this study are shown in Table I. In order to study the effect of the amount of multiplexing on the detector performance, five different multiple pinhole configurations were simulated, with 9, $25,49,121$, and 225 pinholes, respectively. The corresponding pinhole distances were $3.0,2.0,1.5,1.0$, and $0.6 \mathrm{~cm}$. The multiple pinholes were placed in square patterns. The actual pinhole positions were randomized by a small amount (1-2 $\mathrm{mm}$ ) around the grid points to reduce the possible artifacts, which may arise as a result of sampling the object at some discrete spatial frequencies. In these studies, the aperture penetration is modeled by calculating the path length of gamma rays inside the aperture volume. Two such detectors were placed above and below the object. For simulating the penetration effect on the knife-edge of the pinholes, we developed a ray tracing algorithm that first divides the aperture into small volumes and then the total path length is derived based on the number of small volumes went through by the gamma ray and incident angle. An interesting approach for modeling

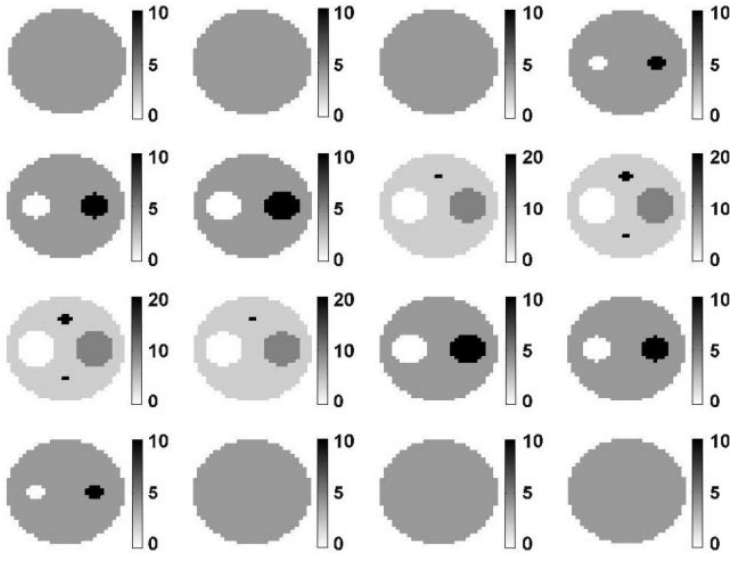

Fig. 2. The phantom used in 3-D study.

the aperture penetration was proposed by Metzler et al. that provides an analytical expression which is useful for speeding up the calculation of the system response function [10], [11].

Due to the large number of detector bins and source voxels involved, it is impractical to precalculate and store the entire system response function (SRF). Instead, we precalculated and stored several sub-SRFs corresponding to factors such as collimator transfer function, probabilities of Compton scattering at every possible angle and detection after scattering, etc. The data were generated in list-mode and SRF corresponding to a particular data realization was precomputed and stored in memory for reconstruction. This precomputation is very time consuming. It also limits the total number of counts that can be reconstructed to less than 1 million. The object simulated was assigned an activity distribution as shown in Fig. 2. It contains four hot or cold spheres, superimposed on a continuous background.

\section{B. Variance-Resolution Tradeoff}

In nuclear medicine, reconstructed images are normally biased estimators of the true objects, with the presence of statistical noise and imperfections in the system model. Therefore, it is important to compare detector performance or image quality as a function of the bias. Barrett et al. proposed to use the linear discrimination of a known signal against a known background as a figure-of-merit [10], [11]. However, most current studies are 
based on some very simple and somehow unrealistic imaging tasks. It has been shown that the simplicity of these tasks limited their use in optimizing system design. Uniform Cramer-Rao bound (Uniform CR bound) has been used to study the variance-bias tradeoff of an estimator [12], [13]. It gives the lowest possible variance at a certain bias described by the bias gradient. This method requires the inversion of the related Fisher information matrix (FIM) and therefore a huge computational load for a relatively complicated detector system.

Fessler and Rogers proposed an approach that analyzes the mean, variance, and spatial resolution properties of images estimated through optimizing an implicitly defined object function, such as the penalized likelihood or maximum a priori (MAP) estimators [14], [15]. This method was used in developing a preconditioner for conjugate gradient PET image reconstruction [16]. Qi et al. further explored this idea by adopting this preconditioner as an approximation of the inverse of the Hessian matrix and developed a closed form approximation for resolution and variance. This later development utilized Fourier transform theory and the assumption that the system modeled are "locally shift-invariant" [17], [18]. It has been shown that the variance and resolution derived had very good agreement with the Monte Carlo simulations. Another important feature about this approach is that it does not require the inversion of the Hessian matrix. Therefore the computation load is greatly reduced. In this study, we adopted this approach to demonstrate the benefit of adding the Compton scatter aperture.

One could easily argue that the spatial resolution and variance properties derived using this approach are only based on a particular of reconstruction algorithm. The arguments we would use to support this choice are the following. Theoretically, it has been shown that the uniform CR bound can be achieved by MAP reconstruction with a quadratic prior [12]. Practically, this is a method that we have relatively comprehensive theoretical understanding and experimental verification. It also requires only modest computational resources, which makes it attractive for our study.

The details of the development leading to this method can be found in the references cited above. Here we briefly restate some of the key steps. Given a measured data set $\boldsymbol{y}$, the log-likelihood of an estimator $x$, of the underlying object is

$$
L(\boldsymbol{y} \mid \boldsymbol{x})=\sum y_{i} \log \bar{y}_{i}-\bar{y}_{i}
$$

where $\boldsymbol{x} \in R^{n}$ is the unknown image and $\boldsymbol{y} \in R^{m}$ is the measured data. The mean of the data is related to the image $x$ through transformation

$$
\bar{y}=\boldsymbol{P x}+\boldsymbol{r}
$$

where $\boldsymbol{P}$ is the detector response function and $r$ is the mean contribution from object scattering events and background radiation. The MAP estimate is achieved by maximizing an object function

$$
\boldsymbol{\Phi}(\boldsymbol{x}, \boldsymbol{y})=L(\boldsymbol{y} \mid \boldsymbol{x})+\beta R(\boldsymbol{x})
$$

where $\beta$ is the parameter that controls the amount smoothing applied to the image. For simplicity, we only used the quadratic roughness penalty with the form

$$
\begin{aligned}
\boldsymbol{R}(\boldsymbol{x}) & =\sum_{j} \phi_{j}\left([\boldsymbol{C} \hat{\boldsymbol{x}}-\boldsymbol{m}]_{j}\right)=\sum_{j} \frac{1}{2} \sum_{i} w_{i j} \phi\left(x_{j}-x_{i}\right)(4) \\
\phi(t) & =\frac{t^{2}}{2}
\end{aligned}
$$

where $w_{i j}$ is the weighting factor. $\boldsymbol{C}$ is the neighborhood matrix whose definition can be found in [17] and $\boldsymbol{m}$ is an arbitrary vector.

So that the MAP estimator is defined as

$$
\hat{\boldsymbol{x}}=\arg \max _{x} \boldsymbol{\Phi}(\boldsymbol{x}, \boldsymbol{y})
$$

For a nonlinear estimator, one can use the local impulse response (LIR) as a measure of the spatial resolution property. For the $j$ th voxel it is defined as

$$
l^{j}(\hat{\boldsymbol{x}})=\lim _{\delta \rightarrow 0} \frac{E\left(\boldsymbol{y}\left(\boldsymbol{x}+\delta \boldsymbol{e}_{j}\right)\right)-E(\boldsymbol{y}(\boldsymbol{x}))}{\delta}=\frac{\partial}{\partial x_{j}} \hat{\boldsymbol{x}}(\boldsymbol{y}(\boldsymbol{x}))
$$

where E[.] is the expectation operator. Using the first order Taylor expansion and chain rule, one can approximate the local impulse response by its linearized representation [21]

$$
l^{j} \approx \frac{\partial}{\partial x_{j}} \hat{\boldsymbol{x}}(\boldsymbol{y}(\boldsymbol{x})) \approx[\boldsymbol{F}+\beta \boldsymbol{R}(\hat{\boldsymbol{x}})]^{-1} \boldsymbol{F} \boldsymbol{e}_{j}
$$

where $\boldsymbol{F}=\boldsymbol{P}^{\prime} \boldsymbol{D}\left[1 / y_{i}\right] \boldsymbol{P}$ is the Fisher information matrix (FIM). The covariance of MAP reconstruction can be approximated as

$$
\operatorname{Cov}(\hat{\boldsymbol{x}}) \approx[\boldsymbol{F}+\beta \boldsymbol{R}]^{-1} \boldsymbol{F}[\boldsymbol{F}+\beta \boldsymbol{R}]^{-1} .
$$

By using the recipe presented in [14], one can calculate the local impulse response function and variance at a certain point through calculating a row of the inversed matrix $[\boldsymbol{F}+\beta \boldsymbol{R}]$.

This method involves the inversion of Hessian matrix or solving related linear equations. For the detector geometry and the 3-D source object used in this study, calculating the FIM (or even a column of FIM) is a very challenging task. To make the calculation computationally practical, we need to make further simplification by assuming the system is "locally shift-invariant" [18]. One can bring the diagonal matrix sandwiched between $\boldsymbol{P}^{\prime}$ and $\boldsymbol{P}$ outside so that

$$
\boldsymbol{F}=\boldsymbol{P}^{\prime} \boldsymbol{D}\left[\frac{1}{\bar{y}_{1}}\right] \boldsymbol{P} \approx \boldsymbol{D}_{\boldsymbol{k}} \boldsymbol{P}^{\prime} \boldsymbol{P} \boldsymbol{D}_{\boldsymbol{k}}
$$

and similarly

$$
\beta R(\hat{x}) \approx \beta D_{k} D_{\eta} C^{\prime} C D_{\eta} D_{k}
$$

where the diagonal matrix $\boldsymbol{D}_{\boldsymbol{k}}=\boldsymbol{D}\left[K_{j}\right]$ with

$$
K_{j}=\sqrt{\frac{\sum_{i} \frac{P_{i j}^{2}}{\bar{y}_{i}}}{\sum_{i} P_{i j}^{2}}}
$$



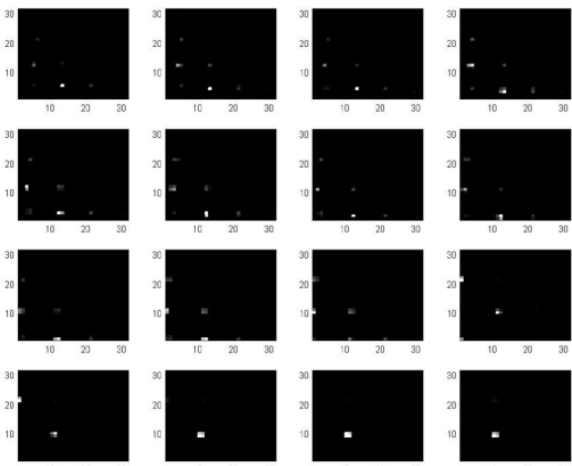

(a): With CSE
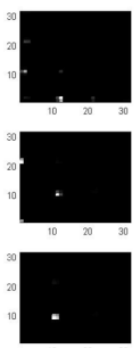
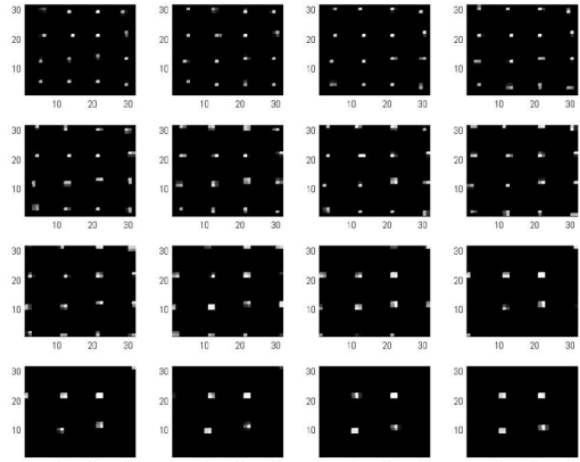

(b): Without CSE

Fig. 3. The probability of a detected event being originated from each source pixel. The brightness of a pixel is proportional to this probability. The central 16 transaxial slices are shown and the 49 pinhole collimator was used in simulation. CSE stands for Compton scattering enhancement and "with CSE" means that the directional information obtained from the detected Compton scattering was included in the image reconstruction.

and $\boldsymbol{D}_{\eta}=\boldsymbol{D}\left[\eta_{j}\right]$ with

$$
\eta_{j}=\sqrt{\frac{\beta}{k_{j}} \frac{\sum_{k} c_{k j}^{2} \ddot{\dot{\phi}}([\boldsymbol{C} \hat{\boldsymbol{x}}-\boldsymbol{m}])}{\sum_{i} c_{k j}^{2}}}
$$

where $\ddot{\phi}(t)=d^{2} / d t^{2} \phi(t)$. Substituting (10) and (11) into (8), one gets the approximation for the local impulse response

$$
l^{j} \approx\left[\boldsymbol{P}^{\prime} \boldsymbol{P}+\eta_{j}^{-2} \boldsymbol{C}^{\prime} \boldsymbol{C}\right]^{-1} \boldsymbol{P}^{\prime} \boldsymbol{P} \boldsymbol{e}_{j} .
$$

Using the locally shift-invariant approximation, the eigenvalues, $\lambda_{i}$ and $\mu_{i}$, of the block-circulant representations of $\boldsymbol{P}^{\prime} \boldsymbol{P}$ and $\boldsymbol{C}^{\prime} \boldsymbol{C}$ can be derived using the 3-D Fourier transform. This property was used to develop closed form expressions for the LIR and variance of MAP reconstruction [17]

$$
\begin{aligned}
l^{j}(\hat{\boldsymbol{x}}) & =\boldsymbol{Q}^{\prime} \boldsymbol{D}\left[\frac{\lambda_{i}}{\lambda_{i}+\eta_{j}^{2}(\hat{x}) \mu_{i}}\right] \boldsymbol{Q} \boldsymbol{e}_{j} \\
\operatorname{Var}_{j}(\hat{\boldsymbol{x}}) & \approx \frac{k_{j}^{-2}}{N} \sum_{i} \frac{\lambda_{i}}{\left(\lambda_{i}+\eta_{j}^{2}(\hat{x}) \mu_{i}\right)^{2}}
\end{aligned}
$$

where $\boldsymbol{Q}$ and $\boldsymbol{Q}^{\prime}$ are the unitary 3-D discrete Fourier transform operator and its transpose. The $j$ th element of the LIR is defined as contrast recovery coefficient (CRC).

One difficulty in using resolution-variance tradeoff for detector optimization is defining the spatial resolution. Many quantities, such as full width at half maximum (FWHM), full width at one-tenth maximum (FWTM), and contrast recovery coefficient (CRC) have been used in the past. However, none of them can fully quantify the spatial resolution property in reconstructed images. In this study, we choose to use CRC as a resolution index based on its simplicity and known correlation to FWHM. It can also be related to the ability of quantifying the activity concentrations in preset regions-of-interest (ROIs) and lesion detection tasks, through the quantity "point-wise signal to noise ratio" [17]

$$
S N R=\frac{c r c_{j}}{\sqrt{v a r_{j}} \cdot \frac{1}{\bar{x}_{j}}}=\frac{c c r_{j} \cdot \bar{x}_{j}}{\sqrt{v a r_{j}}} .
$$

\section{RESULTS}

\section{A. Reconstructions in 3-D}

Fig. 3 shows the back-projected probability distribution across each of the 16 slices of the source object for a detected event. It is easily seen that the angular ambiguity is greatly reduced by adding the Compton scattering information. This was translated into an improved image quality as shown in Figs. 4-6. The list-mode MAP algorithm with quadratic penalty function [as shown in (4) and (5)] was used for these reconstructions. All three data sets contained the same number of counts. Using the Compton scattering information significantly reduced the variance at the same value of CRC. It also helped to achieve a better reconstruction in the cold region. This should improve the accuracy of quantifying the activity concentration within a preset region-of-interest. When comparing the detector performances based on the same measuring time, the detector, using only Compton scattered events, suffered from the low sensitivity and produced the highest standard deviation at the same CRC (resolution). This can be improved if one also makes use of the non-Compton events. These results are shown in Table II.

\section{B. Resolution-Variance Tradeoff}

To further evaluate the proposed detector design, we compared the image quality using the variance-resolution tradeoff curve achieved at the image center. This study took the following steps. First, we compared the relative detector performance as a function of the amount of multiplexing in the data, with collimators having different pinhole configurations. Second, based on the results achieved, we compared the "best" CSE detector (amongst the detectors compared) with the "best" standard MPH detector to show the benefit of combining Compton aperture with mechanical collimation. Finally, we briefly discussed results from resolution-variance trade-off study and highlighted the limitations of this approach.

The effect of the amount of multiplexing on the CSE detector performance was studied using the five multiple pinhole configurations (see Section II). Standard deviations as a function of resolution (CRC) were derived using (15) and (16). The collected data sets were normalized to both same counts and same 

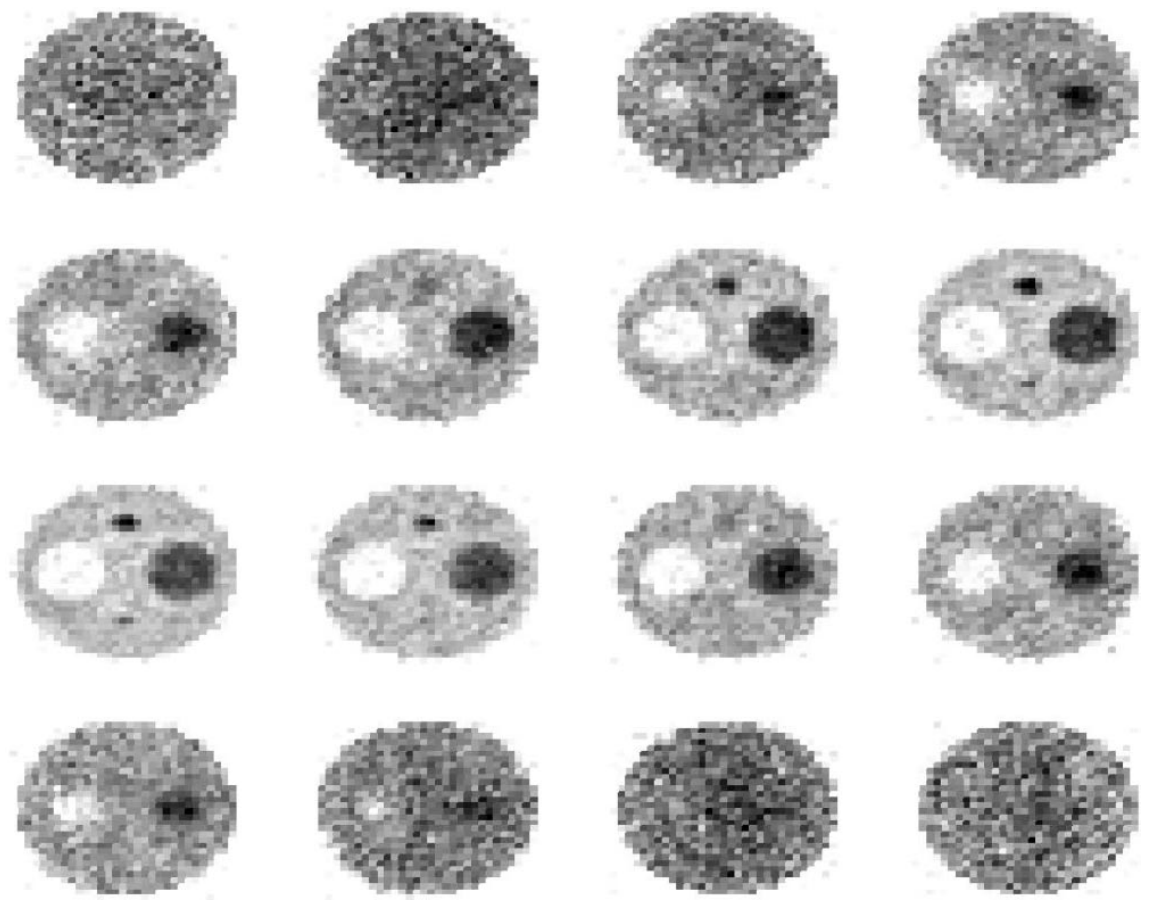

Fig. 4. Reconstructed image with $250 \mathrm{k}$ Compton scattered events. The CRC at the center is 0.3 . The collimator used has 49 pinholes.
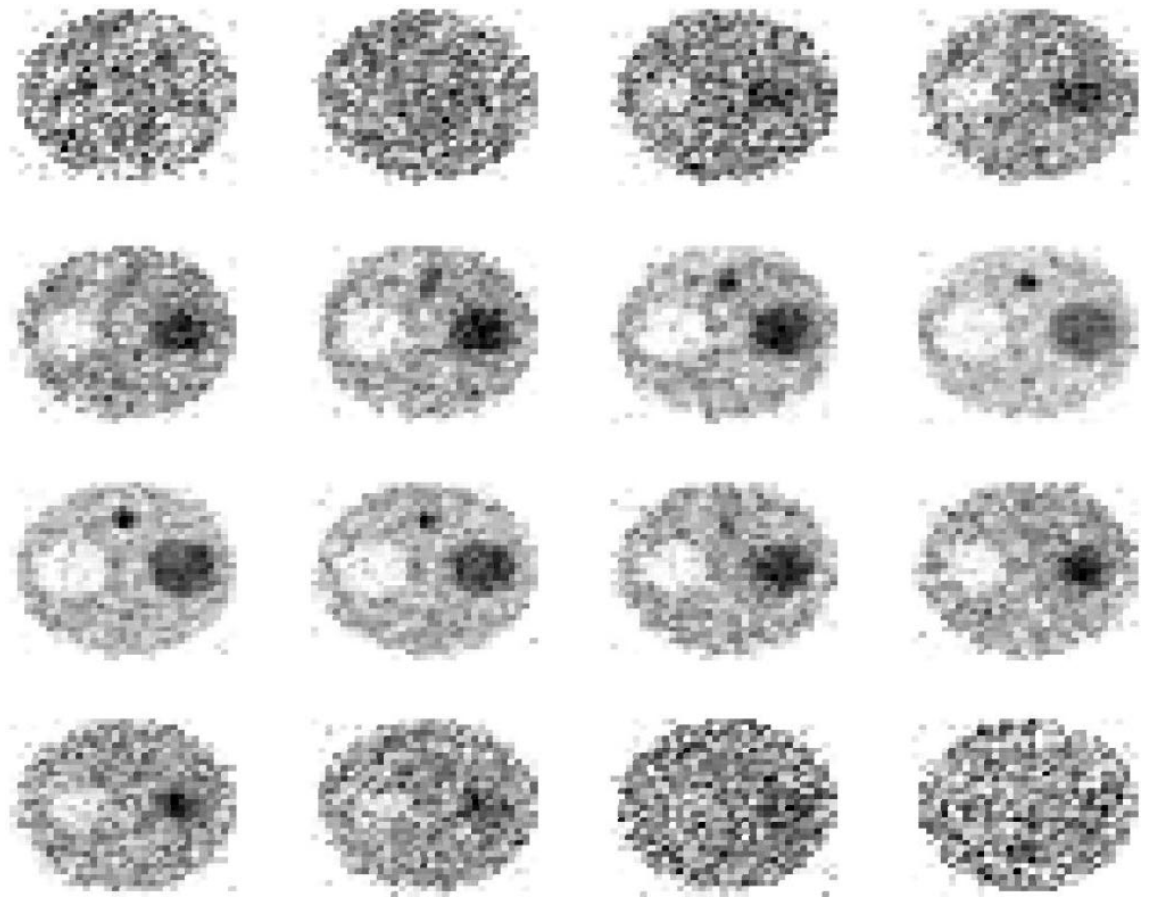

Fig. 5. Reconstructed image with $250 \mathrm{k}$ events, including both Compton scattered and non-Compton scattered events. The CRC at the center is 0.3 . The ratio of Compton scattered events used is determined based on the proposed detector design (see Table I for details).

measuring time and shown in Figs. 7-10. For the standard MPH detector, the best variance-resolution (CRC) was achieved with the 25 pinhole collimator, given the same measuring time. The difference between detectors using the 25 pinhole and 9 pinhole collimators is very small. This indicates that a collimator with relatively small amount of multiplexing is preferred if no extra information is available. When Compton information is added, a larger open fraction on the collimator provided images with the lowest variance. The best variance-CRC (resolution) tradeoff was achieved with the 121 pinhole collimator. It is worth noting that the standard deviation versus CRC curves may go across each other when CRC is relatively large. This is due to changes in the shape of LIRs, which will be discussed later in this section. Fortunately, all useful reconstructions were achieved when CRC is between 0.2 and 0.6. Therefore, we used the curves within this range for comparing the performances of detectors.

To demonstrate the benefit of the proposed detector design, we compared the performances of the "best" CSE detector (using the 121 pinhole collimator) with the "best" standard 

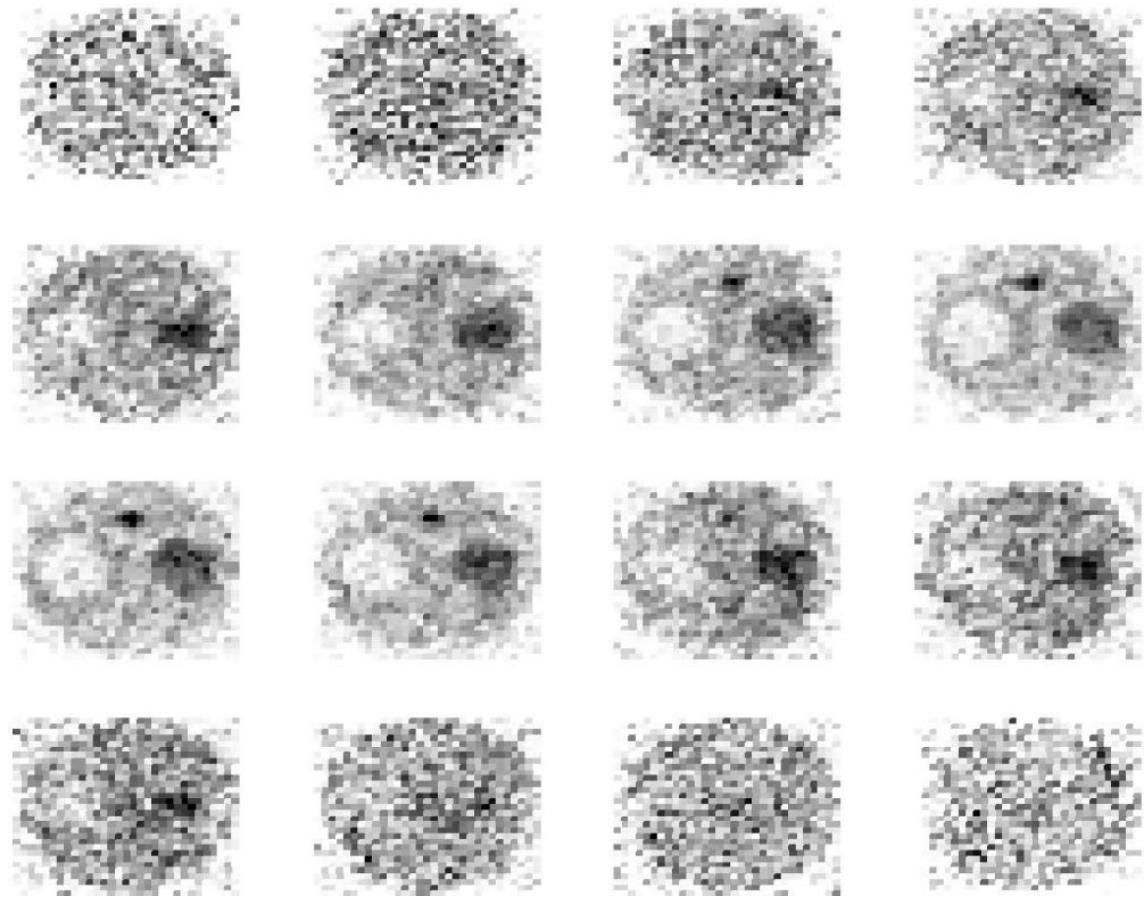

Fig. 6. Reconstructed image with $250 \mathrm{k}$ multiple pinhole events (with no Compton scattering information) events.

TABLE II

IMAGE PROPERTIES AT THE CENTRE OF THE RECONSTRUCTED IMAGES FOR Detectors Using THE 49 PinHOle Collimator

\begin{tabular}{|c|c|c|c|}
\hline & MPH only & CSE+MPH & CSE only \\
\hline CRC & 0.3 & 0.3 & 0.3 \\
\hline $\begin{array}{c}\text { Norm. STD } \\
\text { (Same Counts) }\end{array}$ & 1.0 & 0.61 & 0.42 \\
\hline $\begin{array}{c}\text { Norm. STD } \\
\text { (Same Time) }\end{array}$ & 1.0 & 0.78 & 1.19 \\
\hline
\end{tabular}

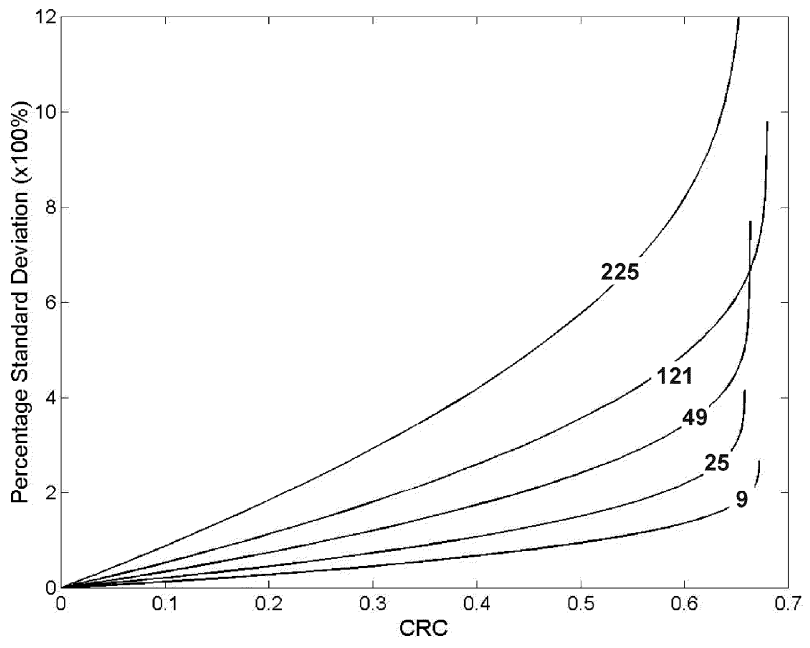

Fig. 7. Standard deviation as a function CRC for detector using standard MPH events (no Compton scattering information used at all). Five MPH configurations were used and curves are normalized to the same counts.

MPH detector using (the 25 pinhole collimator). The results are shown in Fig. 11. It is easily seen that using Compton scattered events only gave the worst performance amongst

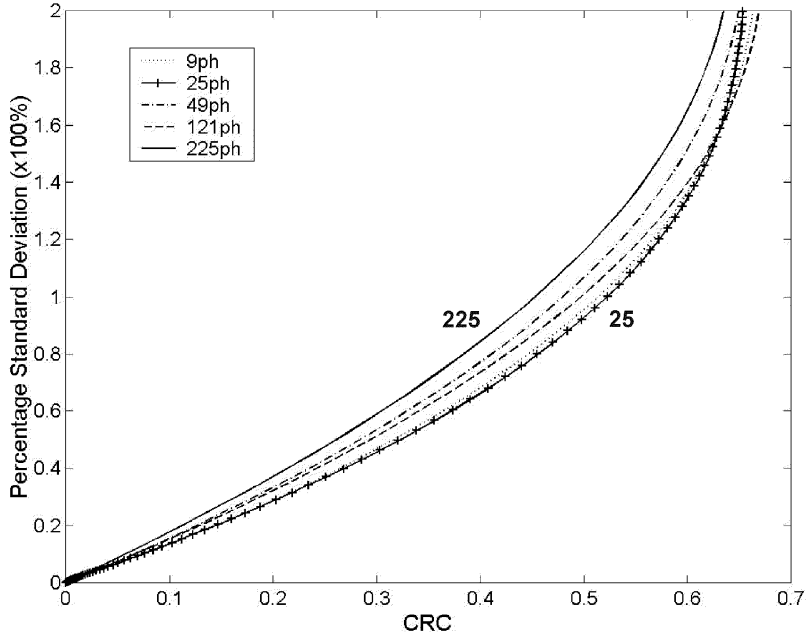

Fig. 8. Standard deviation as a function CRC for detector using standard MPH events (no Compton scattering information used). Five MPH configurations were used and curves are normalized to the same measuring time.

the configurations in the sense that it results in the highest variance at the same CRC. This is due to the relatively low sensitivity of the proposed detector. This limitation can be greatly reduced if one also uses the non-Compton events. In practice, the sensitivity for Compton scattering events may also be improved by having thicker Si detector and better angular coverage for the scattered photons. These may further improve the performance of the proposed detector.

It is worth noting that controlling the CRC to be identical did not result in LIRs with identical FWHM (see Table III). Although the process of fitting the LIR into Gaussian shape may introduce some error, there is another cause for this effect. The shape of actual LIRs changes with detector configuration. Without Compton scattering information, the LIR tends to 


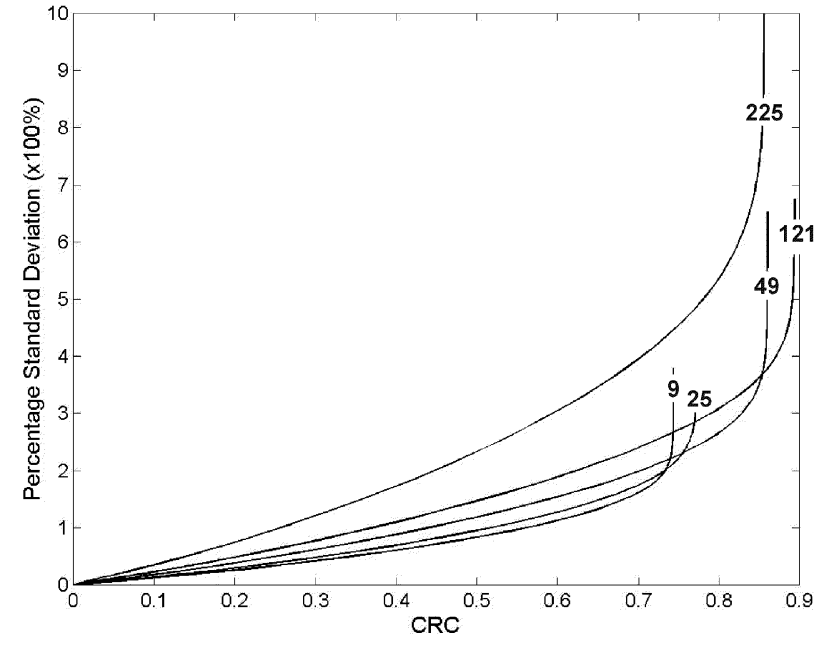

Fig. 9. Standard deviation as a function CRC for detector using 100\% CSE events. Five MPH configurations were used (number on curves indicates the number of pinholes in the collimator) and curves are normalized to the same counts in the data.

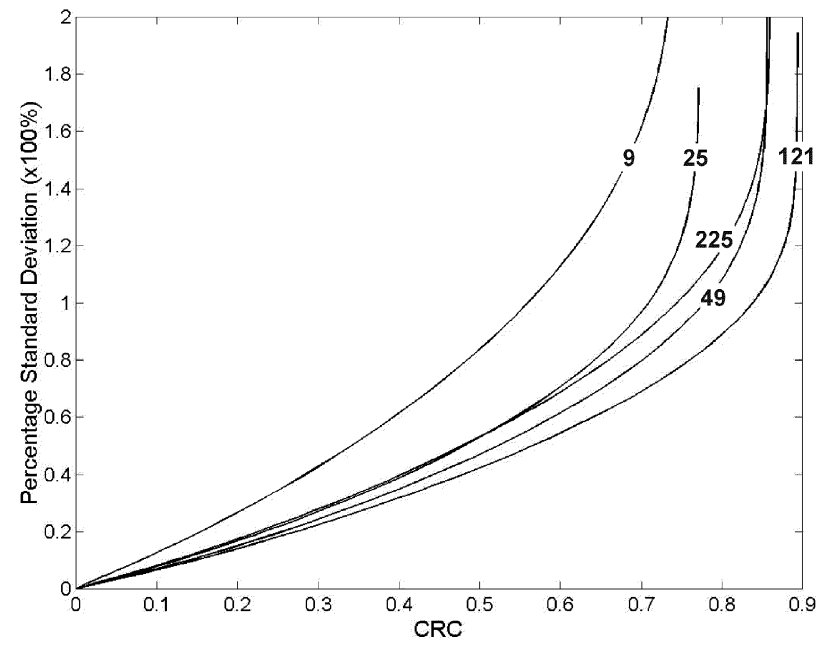

Fig. 10. Standard deviation as a function CRC for detector using 100\% CSE events. Five MPH configurations were used and curves are normalized to the same measuring time.

spread wider to the entire source object space. Therefore the energy left within the region surrounding the peak is reduced. To keep the CRC value unchanged, the FWHM of the LIR need to be reduced. These results provided some interesting indications about the limitations of this approach.

- Using CRC (or more precisely, any single-variate index, including FWHM and FWTM) in resolution-variance tradeoff study could lead to biased results because it can not fully represent the point-spread function.

- The change in the shape of the LIR implies differences in the so-called "noise structure" or covariance in the images. This is not taken into account in the resolution-variance tradeoff studies. Other detector performance indices, such as observer performance may be desired.

For example, comparing variance at a given resolution (CRC) leads to the conclusion that a 25 pinhole MPH detector is better than the 121 pinhole CSE detector using only Compton scattered events. However, both reconstructed images (Fig. 12) and

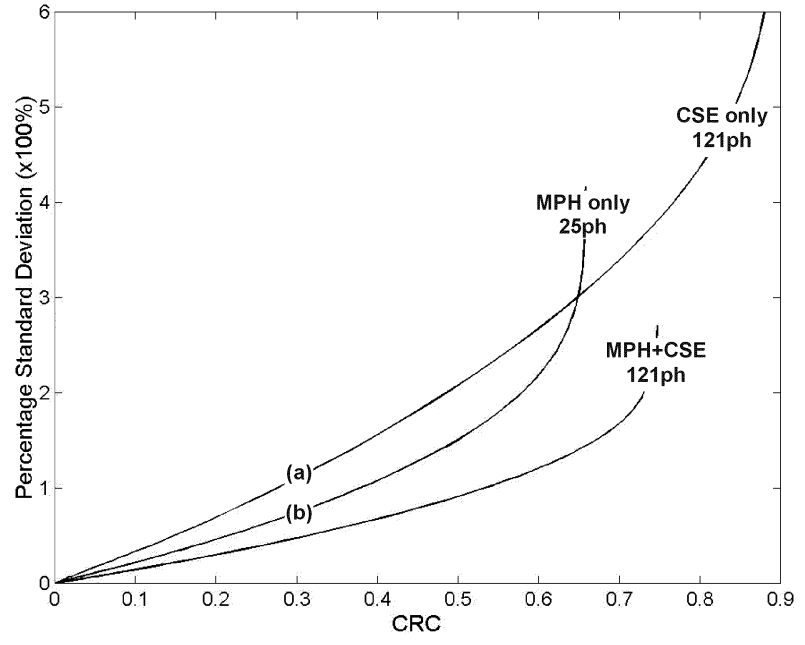

Fig. 11. Comparison between the "best" MPH and CSE detectors.

TABLE III

IMAGE PROPERTY AT THE CENTER OF THE RECONSTRUCTED IMAGE

\begin{tabular}{|c|c|c|c|}
\hline & MPH only & CSE+MPH & CSE Only \\
\hline CRC & 0.3 & 0.3 & 0.3 \\
\hline FWHM & 1.02 pix. & 1.05 pix. & 1.10 pix. \\
\hline $\begin{array}{c}\text { Percentage } \\
\text { Stand. Dev. }\end{array}$ & 0.75 & 0.52 & 1.12 \\
\hline
\end{tabular}

corresponding LIRs (Fig. 13) showed that the latter suffers much less from noise propagation. Although with higher variance, the reconstruction has better reproduction of the low-activity region and attractive visual appearance. It is, therefore, important to optimize the detector performance using criteria that take into account both variance and covariance in the reconstructed images.

\section{CONCLUSION AND DisCUSSIONS}

We presented a comparative study between the standard multiple pinhole detector and the Compton-scattering-enhanced multiple pinhole detector. The results achieved are summarized as follows.

- The proposed CSE detector design results in an improved imaging performance compared with standard MPH detectors.

- It provides not only lower variance but also lower covariance in the image. This should be beneficial for both detection and quantification tasks.

- In order to compensate for the low probability of detecting Compton scattered events, one needs to use collimator with relatively large open fraction and make use of those non-Compton events.

This study also showed that using the resolution and variance expressions (15) and (16) provided a fast and practical way for multivariate detector optimizations. Further improvement may be needed in reducing the approximation used and therefore making the approach more robust. One approach has been proposed by Stayman and Fessler along this line [19]. It removes one of the most suspicious approximations, namely, moving the 

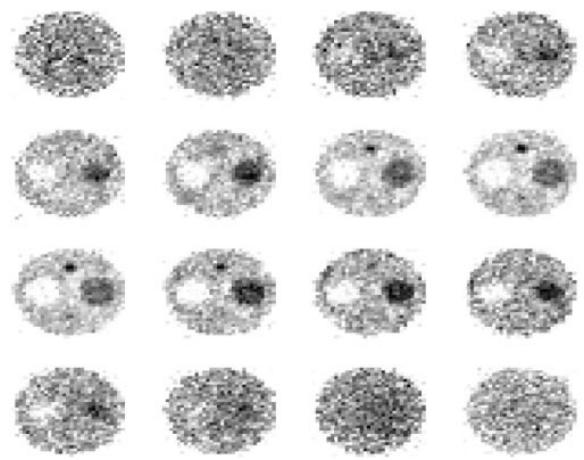

(a)
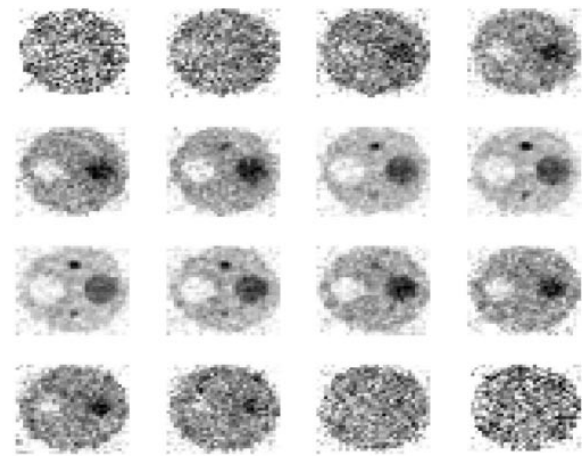

(b)

Fig. 12. Comparing reconstructed image with data collected using (a) the 121 pinhole CSE detector and (b) 25 pinhole MPH detector. The same measuring time was used and CRC was kept to 0.3 at image center for both images. The operating points for both reconstructions are shown in Fig. 11.
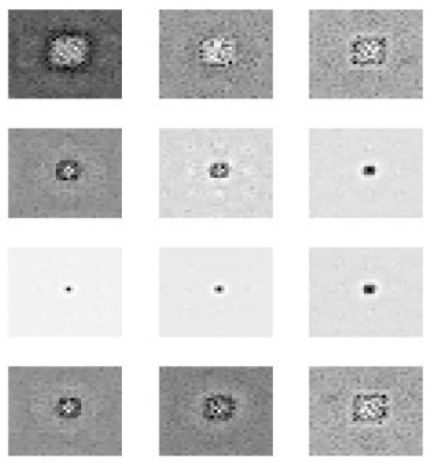

(a)

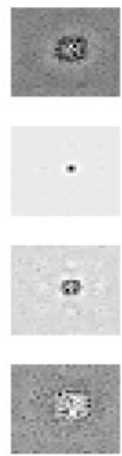

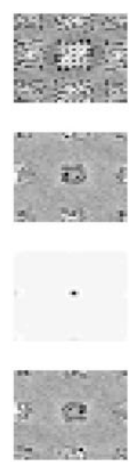
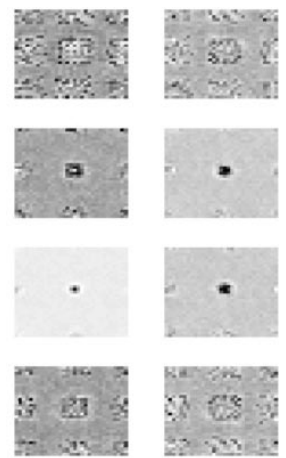

(b)

Fig. 13. Comparing the LIRs at image center for (a) the 121 pinhole CSE detector and (b) 25 pinhole MPH detector. The same measuring time was used and CRC was kept to 0.3. The operating points for both reconstructions are shown in Fig. 11.

diagonal matrix $\boldsymbol{D}\left[1 / y_{i}\right]$ out of the FIM [see (10)]. It is also possible to use the Monte Carlo integration techniques [20], [21] to calculate the FIM. When the calculation involves a complicated detector system, this approach would be a valuable alternative.

The results presented also highlighted the limitations of using variance-resolution tradeoff for detector optimizations. Correlating the results from this calculation with that from task-based observer study is a topic worth effort, because the later also takes into account the covariance or so called "noise structure" in the image. It has been shown that the chanalized hotelling observer (CHO) [22], [23] can also be related to human observer response. Therefore, it would be highly desirable to compare detector performances using $\mathrm{CHO}$ study if one can incorporate a more realistic imaging task.

\section{REFERENCES}

[1] B. M. W. Tusi and G. T. Gillberg et al., "Design and clinical utility of a fan beam collimator for SPECT imaging of the head," J. Nucl. Med., vol. 27, pp. 810-819, 1986.

[2] R. J. Jaszczak and C. E. Floyd et al., "Cone beam collimation for single photon-emission computed-tomography," Med. Phys. Biol., vol. 37, pp. 507-534, 1992.

[3] H. H. Barrett and W. Swindell, Radiological Imaging. New York: Academic, 1981, ch. 8.

[4] S. R. Meikle and R. R. Fulton et al., "An investigation of coded aperture imaging for small animal SPECT," IEEE Trans. Nucl. Sci., vol. 48, pp. 816-821, June 2001.

[5] J. W. LeBlanc and N. H. Clinthorne et al., "C-SPRINT: a prototype Compton camera system for low energy gamma ray imaging," IEEE Trans, Nucl. Sci., vol. 45, pp. 943-949, June 1998.
[6] C. H. Hua, N. H. Clinthorne, S. J. Wilderman, J. W. LeBlanc, and W. L. Rogers, "Quantitative evaluation of information loss for Compton cameras," IEEE Trans. Nucl. Sci., vol. 46, pp. 587-593, June 1999.

[7] A. Uritani, N. H. Clinthorne, and J. E. Gormley, "Electronically-collimated gamma camera with a parallel plate collimator for Tc-99 m imaging," IEEE Trans. Nucl. Sci., vol. 44, pp. 894-898, June 1997.

[8] J. Qi, R. M. Leahy, S. R. Cherry, A. Chatziioannou, and T. H. Farquhar, "High resolution 3D Bayesian image reconstruction using MicorPET small animal scanner," Phys. Med. Biol., vol. 43, no. 4, pp. 1001-1013, 1998.

[9] J. Y. Qi, R. M. Leahy, C. Hsu, T. H. Farquhar, and S. R. Cherry, "Fully 3D Bayesian image reconstruction for ECAT EXACT HR+," IEEE Trans. Nucl. Sci., vol. 45, pp. 1096-1103, June 1998.

[10] S. D. Metzler, J. E. Bowsher, K. L. Greer, and R. J. Jaszczak, "Analytic determination of the pinhole collimator's point-spread function and rms resolution with penetration," IEEE Trans. Med. Imaging, vol. 21, pp. 878-887, Aug. 2002.

[11] H. H. Barrett, J. Denny, R. Wagner, and K. Myers, “Objective assessment of image quality II: Fisher information, Fourier crosstalk and figure of merit for task performance," J. Opt. Soc. Amer. A, vol. 12, pp. 834-852, 1995.

[12] A. O. Hero, J. A. Fessler, and M. Usman, "Exploring estimator biasvariance tradeoffs using the uniform CR bound," IEEE Trans. Signal Processing, vol. 44, pp. 2026-2041, Aug. 1996.

[13] A. Hero and J. A. Fessler, "A recursive algorithm for computing Cramer-Rao-type bounds on estimator covariance," IEEE Trans. Info. Theory, vol. 40, pp. 1205-1212, 1994.

[14] J. A. Fessler, "Mean and variance of implicitly defined biased estimator (such as penalized maximum likelihood): Application to tomography," IEEE Trans. Image Processing, vol. 5, pp. 493-506, Mar. 1996.

[15] J. A. Fessler and W. L. Rogers, "Spatial resolution properties of penalized-likelihood image reconstruction: Space-invariant tomographs," IEEE Trans. Image Processing, vol. 5, pp. 1346-1358, Sept. 1996.

[16] J. A. Fessler and S. D. Booth, "Conjugate-gradient preconditioning methods for shift-variant PET image reconstruction," IEEE Trans. Image Processing, vol. 8, pp. 688-699, May 1999. 
[17] J. Y. Qi, "A theoretical study of the contrast recovery and variance of MAP reconstructions from PET data," IEEE Trans. Med. Imaging, vol. 18, pp. 293-305, 1999.

[18] J. Y. Qi and R. M. Leahy, "Resolution and noise properties of MAP reconstruction for fully 3-D PET,” IEEE Trans. Med. Imaging, vol. 19, pp. 493-506, May 2000.

[19] J. W. Stayman and J. A. Fessler, "Efficient calculation of resolution and covariance for fully-3D SPECT," IEEE Trans. Med. Imaging, vol. 19, pp. 601-615, June 2003.

[20] L. Parra and H. H. Barrett, "List-mode likelihood: EM algorithm and image quality estimation demonstrated on 2-D PET," IEEE Trans. Med. Imaging, vol. 17, pp. 228-235, Apr. 1998.
[21] Numerical Recipes in C, Cambridge Univ. Press, Cambridge, U.K., ch.

[22] H. H. Barrett, T. Gooley, and K. Girodias, "Linear discriminants and image quality," Image Vision Comput., vol. 10, pp. 451-460, 1992.

[23] R. D. Fiete, H. H. Barrett, and W. E. Smith, "Hotelling trace criterion and its correlation with human-observer performance," J. Opt. Soc. Amer. A, vol. 4, pp. 945-953, 1987. 